\title{
OPTICAL PROPERTIES OF FR I RADIO GALAXIES
}

\author{
MICHAEL J. LEDLOW \\ Dept. of Astronomy, New Mexico State University \\ Las Cruces, NM 88003-0001, USA
}

AND

FRAZER N. OWEN

National Radio Astronomy Observatory

Socorro, NM 87801, USA

\section{Introduction}

From the VLA $20 \mathrm{~cm}$ survey of $\sim 500$ Abell clusters reported by (Ledlow and Owen, 1995), we have obtained optical R-Band CCD observations and optical spectra for 265 radio galaxies. The survey is complete for $20 \mathrm{~cm}$ flux density greater than $10 \mathrm{mJy}$ within 0.3 corrected Abell radii of the cluster center. All Abell clusters with measured $z<0.09$ were surveyed. This statistically complete sample was supplemented by $\sim 200$ clusters with $0.09<z<0.25$ including sources with flux density $>200 \mathrm{mJy}$. Only $6 \%$ of the sample consists of FR II radio sources, the remainder are twin-jets, tailed, or compact sources associated with the FR I class.

Using optical surface photometry, we have examined the surface brightness profiles, sizes, ellipticities, nuclear luminosities, and isophotal properties of these rich cluster radio galaxies. The frequency of galaxy-galaxy interactions, close companions, and distorted isophotes was also examined. A control sample of 50 radio-quiet ellipticals chosen from the same cluster fields was constructed and analyzed identically. From the surface photometry and optical spectra, we ask the question: Are radio sources found in a unique population of elliptical galaxies? If so, what distinguishes radio-loud from radio-quiet galaxies?

From the statistically complete sample for $z<0.09$, we have constructed the univariate radio and bivariate radio/optical luminosity functions. From these functions we examine how the optical properties of the host galaxy influence radio source lifetimes and evolution. We also compare the lumi- 
nosity functions for our rich-cluster sample to the non-rich cluster radio galaxy samples from (Auriemma et al., 1977) and (Sadler et al., 1989) to explore the effects of the local galaxy density and environment on radio detection statistics.

\section{Conclusions}

From the described analysis we make the following conclusions:

- The cluster richness and optical morphology do not affect the radio galaxy detection statistics. The number of radio galaxies simply scales with the number of galaxies surveyed.

-Radio Galaxies trace the normal elliptical galaxy optical luminosity function.

-While there is no strong correlation between optical and radio luminosity, the division between FR I and FR II sources does depend on the optical luminosity $\left(\propto L_{\text {opt }}^{2}\right)$.

-The surface-brightness profiles, nuclear luminosities, fundamental plane, luminosity/size relationship, and galaxy shape are not significantly different for radio-quiet and radio-loud ellipticals at the same optical luminosity. - Only $6 \%$ of FR I's show evidence for galaxy interactions. $25 \%$ show evidence of optical peculiarities such as twisted, non-concentric, or non-elliptical isophotes. However, these statistics are identical for radio-quiet ellipticals selected from the same environment. The frequency of companion galaxies ( $<20 \mathrm{kpc}$ projected distance) is the same between the radio galaxy and control sample.

-The break in the differential bivariate luminosity functions corresponds to the FR I/II division and shifts to higher powers as $L_{\text {opt }}^{2}$.

- At a constant radio power, the fraction of galaxies detected increases with optical luminosity. The optical luminosity, or parameters related to it (such as the total mass, ISM density, pressure, and extent) must influence the initial conditions and subsequent evolution of radio sources.

-From both spectroscopy and optical surface photometry, FR I radio galaxies are indistinguishable from radio-quiet ellipticals. Possibly all elliptical galaxies at some time (or many times) have powerful radio sources. This is consistent with the integrated univariate RLF assuming radio source lifetimes are less than a few times $10^{9}$ years.

\section{References}

Auriemma, C.G. et al. 1977, $A \& A, 57,41$.

Ledlow, M.J. \& Owen, F.N. 1995, A.J., 109, 853.

Sadler, E.M., Jenkins, C.R., \& Kotanyi, C.G. 1989, M.N.R.A.S, 240, 591. 